Document downloaded from:

http://hdl.handle.net/10251/34804

This paper must be cited as:

Heras Barberá, SM.; Atkinson, KM.; Botti Navarro, VJ.; Grasso, F.; Julian Inglada, VJ.; Mcburney, PJ. (2013). Research opportunities for argumentation in social networks.

Artificial Intelligence Review. 39(1):39-62. doi:10.1007/s10462-012-9389-0.

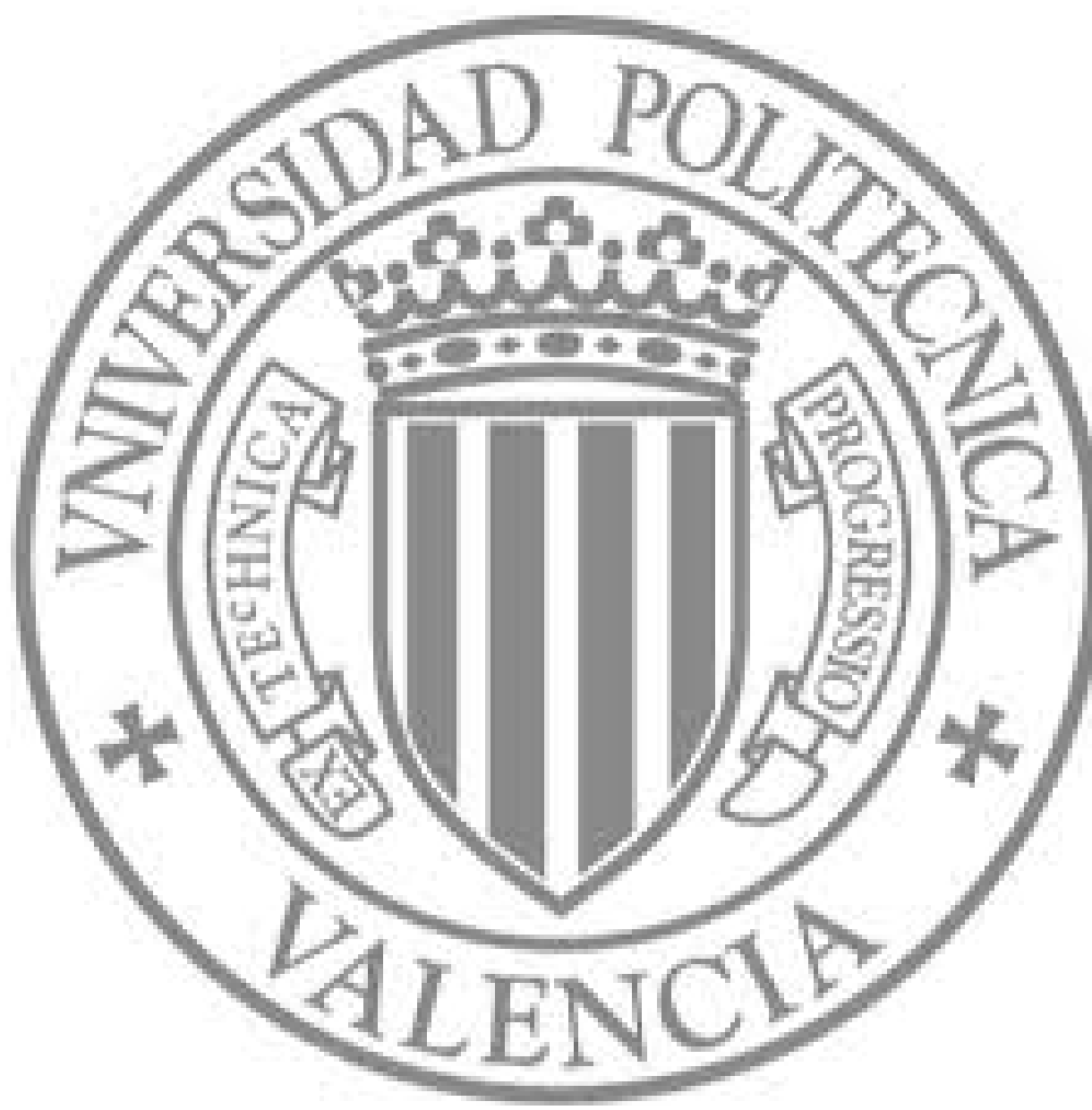

The final publication is available at

http://link.springer.com/article/10.1007\%2Fs10462-012-9389-0

Copyright Springer Verlag 
Noname manuscript No.

(will be inserted by the editor)

\title{
Research Opportunities for Argumentation in Social Networks
}

\author{
Stella Heras • Katie Atkinson • Vicente Botti • \\ Floriana Grasso • Vicente Julián • Peter McBurney
}

Received: date / Accepted: date

\begin{abstract}
Nowadays, many websites allow social networking between their users in an explicit or implicit way. In this work, we show how argumentation schemes theory can provide a valuable help to formalize and structure on-line discussions and user opinions in decision support and business oriented websites that held social networks between their users. Two real case studies are studied and analysed. Then, guidelines to enhance social decision support and recommendations with argumentation are provided.
\end{abstract}

Keywords Social Networks · Customer Support · Recommendations · Argumentation

\section{Introduction}

Currently, the Web has evolved from read-only HTML documents and personal websites to social sites where people interact, share and constantly update huge amounts of decentralised information. This new conception of the Web as a platform for computing and collaborative interaction has been supported by the development of so-called Web 2.0 technologies and standards like AJAX, SVG, FOAF, PHP, Ruby, XHTML, P2P or XSL. The result has been the fast proliferation of web-based communities, on-line social networks, web applications and webservices.

Nowadays, many websites allow social networking between their users in an explicit or implicit way. While some are declared leisure oriented social networking sites, others are more decision support or business oriented while still allowing their users to interact, share their preferences and profiles, form communities with other users and give advice, recommendations and feedback about their experiences. Some examples of the former type are Facebook, Flickr, MySpace, Orkut and Twitter, whose general purpose is to keep users in contact by providing them with a smart interface to show their profile, manage their acquaintances list, join groups, share media content and chat. The latter is the case of on-line shopping companies, such as Amazon or eBay and consumer review sites, like Tripadvisor or Epinions, which provide their users with a means of networking and discussing matters related to their business or reviewing topic. In addition, there is a third type of on-line social networking site whose orientation lies between leisure, business and decision support. An example of this further type of websites

Stella Heras · Vicente Botti · Vicente Julián

Departamento de Sistemas Informáticos y Computación, Universitat Politècnica de València

Camino de Vera s/n, 46022, València, Spain

E-mail: \{sheras,vbotti,vinglada\}@dsic.upv.es

Katie Atkinson · Floriana Grasso

Department of Computer Science, University of Liverpool

Ashton St, Liverpool, L69 3BX, UK

E-mail: \{katie,floriana\}@liverpool.ac.uk

Peter McBurney

Department of Informatics, King's College London

Strand, London WC2R 2LS, UK

E-mail: peter.mcburney@kcl.ac.uk 
is Kiva, which support networking and debate to promote ethical values and social help. Regardless of the purpose of the social networking, in all of these communities discussions arise from the difference of opinion between users, and individual views are mixed in the tangle of user-generated content posted in discussion boards, wikis and blogs. Therefore, there is an obvious need for mechanisms to structure this information and to elicit as much useful knowledge as possible from it.

In this paper, we show how Argumentation Theory (concretely Argumentation Schemes Theory [40]) can provide a valuable help to formalize and structure on-line discussions and user opinions ${ }^{1}$. Argumentation schemes are stereotyped patterns of human reasoning that can improve the user's understanding about discussions and provide a means to evaluate what users have stated and why. When opinions are product recommendations to other users, they are usually justified because they match the user profile (i.e. fit the content of user's declared preferences and likes), the profile of similar users (i.e. collaborative filtering) or both (i.e. hybrid recommendations). Usually, there is not an explanation about the reasoning process that has been followed to come up with specific recommendations. In fact, these recommendations tend to come directly from the recommendation algorithm that runs the website and not from the acquaintances that a user has in his social network. However, this does not follow future trends on the Web, where discovering is becoming social (as reported by Joe Kraus, Google's director of product management in a talk at the Supernova conference 2008) and consequently, recommendations could be expected to come directly from acquaintances in a decentralised way. Moreover, people trust recommendations more when the engine can explain why it made them [22] and what is understood as a good recommendation is changing from the one that minimises some error evaluation measure about the output of content, collaborative filtering or hybrid recommendation methods to the one that really makes people happier.

On the other hand, when user opinions are conveyed in reviews and guides that users write to provide pieces of advice to other users, the reasons that the author has put forward his ideas may be implicit in the text. However, for non-expert users identifying and developing a deep understanding about all such implications can be difficult. Moreover, many reviews and guides are written collaboratively between several users, starting with an initial review followed up with many comments and replies. Thus, each individual opinion can be blurred as the number of posts grows.

Regarding evaluation, user opinions are commonly assessed using some measures of trust and reputation (e.g. usefulness degrees, reviewer ranks, seller ratings and user feedbacks) in decision support or business oriented websites. These values are internally computed in the website by providing users with rating tools to score the posts of other users or to leave feedback about their experiences. However, they are not usual on leisure oriented social networking sites, where the truthfulness of the user opinion has a lesser importance since its final consequences do not usually give rise to a wrong decision or an unsuccessful commercial transaction. Nevertheless, even when user opinions are attached with trust and reputation values, these measures do not provide an objective way of assessing them, for instance, by looking at the reasoning patterns that they follow to come up with specific conclusions. Thus, user opinions can be misunderstood and rated low, decreasing unfairly the trust and reputation values of their authors.

In this work, we focus on analysing the advantages of applying argumentation to business oriented websites that allow a social interaction among their users. Although this analysis could have also been performed on decision support, leisure or ethics oriented social networking sites, for the time being we have limited it to this particular type of website, since user opinions and discussions in them have more restricted topics and a specific objective (that of recommending or reporting items and providing feedback and help). Therefore, the scope and target of on-line business websites makes them more interesting and useful with respect to providing tools to analyse opinions and elicit knowledge from their users. Our study is aimed at showing how argumentation can provide a formal structure to users' opinions and act as a tool to justify positions, to better understand others' views and to be able to asses them. Thus, we do not intend to replace trust and reputation measures with arguments that could be evaluated instead, but we try to clarify the reasoning process that have brought about the opinions of each user. Moreover, formalizing with arguments the reasoning behind user opinions not only improves their understanding and eases their evaluation, but also can provide users that have opposing opinions with tools to reply and rebut others' opinions in a structured way.

1 This paper presents an extended and corrected version of the work published at [14] and [15] 
This paper is structured as follows. Section 2 shows related work on argumentation in social networks. Section 3 introduces the social network model that we use in this research. Section 4 shows the analysis performed about a real study case. Section 5 identifies open research opportunities for argumentation in social networks and proposes the application of an argumentation theory to formalise the underlying reasoning shown in the dialogues held among the users of the network. Finally, section 6 summarises this work and points out guidelines for decision support and business oriented websites to improve on-line dialogues in social networks using argumentation.

\section{Related Work}

Generally speaking, argumentation provides users with a powerful way of justifying pieces of advice and recommendations, clarifying the reasoning process that has been followed to come up with the arguments and supporting usual social network analysis measures like trust and reputation. In this section, we review argumentation research related with these and other topics that could be useful to advance the state-of-the-art in social networks.

\subsection{Trust and Reputation}

Although not being directly applied to social networks, the literature reports some interesting works that apply argumentation to support trust and reputation. In [17], a framework for evaluating trust-related arguments in on-line stores is proposed. This research also proposes a methodology to identify trust related issues and performs an empirical evaluation on 10 websites to demonstrate its applicability. In later research, this model has been extended by applying the Toulmin model of argument to provide guidelines for the implementation of well-structured trust-assuring arguments and to investigate if the provision of these arguments actually increases the user trust in Internet stores [18]. In [35], Toulmin proposed a new model of practical argument focused on the justificatory function of argumentation, as opposed to the inferential function of theoretical arguments. Toulmin suggested that a good argument can provide a good justification for a claim that will stand up to criticism and earn a favourable verdict. Elements to analyse arguments in the Toulmin's model are the claim, the evidence, the warrant, the backing the rebuttal and the qualifier.

Also, in [3], a new quantitative trust model for argumentation-based negotiating agents is proposed. In this work, the shortcomings of current approaches of trust to provide a secure environment for agent negotiation within multi-agent systems, particularly in emerging applications like e-business, are addressed by applying a negotiation dialogue game. Furthermore, the authors present a model for securing agent oriented systems in which agents have an argumentation system that provides them with reasoning capabilities over the reputation of other agents. Our approach of applying argumentation to social networks does not intend to provide new measures of trust and reputation for the users of the network, but support the existing measures with arguments that justify them and clarify the reasoning process behind them.

\subsection{Recommender Systems}

Recommender systems is a mature area of research with a wide history of successful developments in many domains, such as in e-commerce applications [31]. Commonly, recommendations in these systems are based on quantitative measures of similarity between the user's profile of preferences and current items to recommend (i.e. content-based recommenders [26]), between the user's profile and the profile of other users with similar preferences (i.e. collaborative filtering recommenders [32]) and on combinations of both approaches (i.e. hybrid recommender systems [5]). Moreover, there are also works that have proposed trust-based recommender systems operating on social networks. Some examples are [38], where the dynamics of trust and the impact of the network topology in the recommendations provided by agents are investigated; [10], which proposes an interface to generate predictive movie recommendations from social trust; [12], which builds a network of trust propagating schemes and evaluates the model on the Epinions social network; and [24], which presents a new opinion-based filtering method that operates 
on a network of friends with a model of social trust. Finally, in [30] the Regret reputation model was extended to take advantage of the social relations conveyed in a social network of agents.

However, a recent study has stated the inability of current recommender systems to use the large amount of qualitative data available on-line to empower recommendations [8]. The authors propose an argumentation approach to cope with this limitation and provide recommender systems with an argumentation formalism based on Defeasible Logic Programming [9] that models users' preferences by means of facts, and strict and defeasible rules. This formalism also features an inference tool to analyse and decide conflicts among the set of possible suggestions (i.e. recommendations) that the user has been presented with. Another recent work that applies argumentation to manage the interaction that emerges from the recommendation dialogues between a social network of agents has been presented in [13]. This research proposes a dialogue game [29] that controls the recommendation process and the use of Argumentation Schemes [40] to define a set of potential attacks to the recommendations provided by agents. Here, the concepts of argumentation, recommender systems and social networks are studied together for the first time.

An important additional contribution of [8] is the identification of a number of research opportunities for the development of new generations of recommenders, such as exposing underlying assumptions behind recommendations, approaching trust and trustworthiness from the perspective of backing arguments and providing rationally compelling arguments for recommendations. Our work involves a contribution in these areas but from a different perspective than the one proposed in [8], that of structuring and clarifying the reasoning process followed by users to provide pieces of advice and recommendations to other users of their social network.

\subsection{Prediction Markets}

Preliminary work on applying argumentation to prediction markets was proposed in [25]. Prediction markets, also known as information markets, are considered a special type of social network whose purpose is to aggregate the information conveyed by their users to make predictions about specific events or parameters. This information comes in the form of individual bets that can be interpreted as the probability that the users assign to the event to occur or to the parameter to have a final value. The work analyses the influence of the social relationships on the predictions made by group judgement, where a group of agents linked via a social network argue on the final outcome of a prediction.

\subsection{Online Argumentation Tools}

Currently, there are several argumentation tools that offer support for on-line debates, with varying degrees of structure given to the arguments. Some examples are Debategraph ${ }^{2}$, an evolution of DebateMapper that includes it as a view to comment, build, edit and rate debates; Debatepedia ${ }^{3}$, a new free wiki encyclopaedia of arguments and debates and the tool for evaluate debate TruthMapping [37]. The most recent developments in this area have introduced Web 2.0 standards to support on-line debate in the Social Semantic Web. Some contributions of this type are Cope_it! [16], which encourages collaboration by sharing opinions and resources; the semantic web-based argumentation system ArgDF [27]; Cohere [4], a web tool for social bookmarking, idea-linking and argument visualisation; the Argument Blogging project [41], which intends to harvest textual resources from the Web and organise them into distributed argumentative dialogues and the On-line Visualisation of Argument (OVA $)^{4}$ tools, which facilitate argument analysis and manipulation in on-line environments. Some examples of tools that are of a more formal and structured nature include the Parmenides system [6] and the Carneades system [11]. Parmenides is an on-line discussion forum that presents political policy justifications in the form of an argument scheme [6], and it allows users to critique the policy proposals through critical questioning intended to pinpoint the parts that users agree or disagree with most. This the system was developed to address some of the issues in using unstructured tools for posting opinions on on-line systems, similarly to the issues that we have identified here in social networks. Carneades is a tool that provides support

\footnotetext{
2 Debategraph: http://debategraph.org/

3 Debatepedia: http://wiki.idebate.org/

4 OVA at ARG:dundee: www.arg.dundee.ac.uk
} 
for a variety of argumentation tasks, including argument construction, evaluation, mapping and visualisation. For a comprehensive and comparative overview of argumentation tools for the Social Semantic Web we refer the reader to the review published at [33]. As pointed out in that work, there is significant potential for supporting argumentation on the Social Semantic Web, but a large amount of work remains to be done, for instance, in creating ontologies, easing human annotation of arguments and improving techniques for detecting and mining argumentation.

\subsection{Argument Interchange Format}

Another challenge of argumentation is how to communicate arguments and represent argumentation dialogues between different users and systems in a way that they can be understood by all parties. Most of the on-line debate systems of the previous section have developed their own formats for argument representation, commonly only distinguishing between premises and conclusions. Therefore, this variety of representations hinders the compatibility among systems and gives rise to a lot of limitations for online argument interchange. Research on a standard format for argument interchange has resulted in the so-called Argument Interchange Format (AIF) [7], which proposes an ontology to represent argumentation concepts. Lately, this ontology has been extended taking into account the potential needs of Web 2.0 mass argumentation tools [28]. This new extension of the AIF ontology allows for the representation of argumentation schemes and is traduced to the $\mathrm{W} 3 \mathrm{C} \mathrm{OWL}^{5}$ language. Therefore, it eases automatic description logic reasoning over argument structures. Another proposal, specifically designed for expressing argumentative discussions in social media sites, is the Semantic Interlinked On-line Communities (SIOC) Argumentation ontology module [20]. The SIOC ${ }^{6}$ initiative provides an ontology to express rich data from the social Web in the Resource Description Framework (RDF) ${ }^{7}$. Used in conjunction with the Friend Of A Friend (FOAF) ${ }^{8}$ vocabulary, it can also represent personal profile and social networking information. The argumentation module captures the semantics of argumentative discussions by using a new argumentation ontology based on the DILIGENT argumentation ontology concepts [34].

\section{Social Network Model}

There are different approaches for the study of social networks. On the one hand, disciplines such as Physics, Mathematics, Information Sciences and Economics are more interested in the study of social network analysis measures and the network topology and structure. On the other hand, modern Sociology is more focused on the study of the emergent social behaviour on the network, social tendencies and autoorganisation issues. In this section we briefly introduce the model of social network that we have followed to perform the analysis shown in next sections.

We consider social networks in a very general way, as an abstraction to represent social structures that link individuals or organisations. These links can stand for different types of interdependency, such as friendship, trade, shared knowledge, common hobbies, etc. Nowadays, many websites allow their users to interact and form what we call explicit social networks. In this case, the website stores information about users and links between them. Therefore, users can access part of this information and for instance, search their contact list to interact with other users. However, there are other websites that provide their users with social networking features that allow them to interact, but afterwards do not explicitly share with them the information that these interactions generate. In this other case, which we call implicit social networks, the website can also store information about the social relationship between its users, but usually they cannot access to contact lists to retrieve previous partners or do not have an easy way of searching reports about previous dialogues, although the website may display them during a certain period of time. For both types, we identify the following concepts that define a social network in our model:

- Overall purpose and domain of the network: e.g. friendship, business, shared hobbies, ...

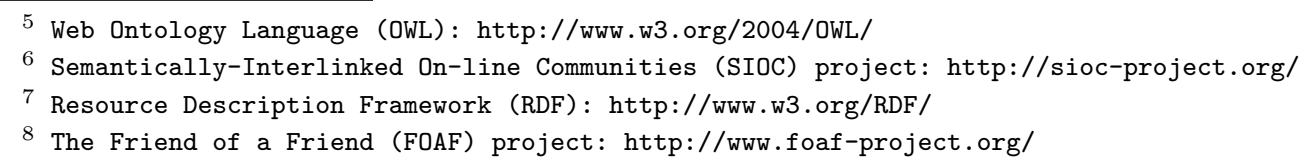


- Tasks Permitted: e.g. recommend things, provide opinions, evaluate others' opinions as witness, evaluate reputation, ...

- Types of dialogues: the different classes of dialogues that can be held in the network (i.e. persuasion, negotiation, inquiry, deliberation, information seeking and sharing and eristics, following the typology in [39]).

- Nodes: represent users (individuals or organisations) playing specific roles.

- Roles: possible roles that individuals or organisations can play in the social network.

- Knowledge databases: we consider that each user of the network has associated individual or shared knowledge databases to represent information about the issues related with each role.

- Ties: links between nodes. There are many different types of ties, depending on the overall purpose of the network (e.g. values, visions, ideas, financial exchange, friendship, personal relationships, kinship, dislikes, conflict, trade) and the tasks permitted.

- Social network analysis measures: we consider that ties and nodes can be labelled with measures to evaluate the relations that they represent. Values of trust and reputation are common examples of these measures, but there may be many others.

Next section provides examples of different social networks in a study case. In the figures that represent these examples, circles stand for nodes (i.e. users playing a role) and lines are the social ties between them. The social ties between users are directed, starting from the node that represents the user that makes the action that generates the tie. In this way, both users can be aware of their social ties and label these ties with different values. However, ties do not necessarily have to be labelled.

\section{Study Case: Amazon}

As pointed out before, our focus of study is business oriented websites that allow a social interaction among their users. Despite their business or support orientation, whose main objective is not that of allowing users to interact in a social network, they still offer social networking features to their users. However, the advantages that the application of argumentation techniques can provide in them are potentially more interesting and obvious than in leisure oriented websites. For instance, argumentation can enhance the benefits of the commercial transactions or the degree of user satisfaction with the recommendation received and hence, the reputation, success and profitability of the company running the website. For performing our study, we have selected a real example of business oriented websites, Amazon, which offers a wide range of social networking features to their users. These features include those typically offered by leisure and support oriented social networking websites. Therefore, the analysis performed over this example is also suitable for a wide range of websites that provide their users with the same social networking features. This section shows the results obtained from the study performed on the example, which also applies to similar websites. Then, the advantages of applying argumentation and specifically, argumentation schemes theory to on-line dialogues in Amazon will be presented in section 5 .

\subsection{Social Networking Features}

Amazon is the largest on-line retailer of USA offering sales on a wide range of products, from books, CDs and DVDs to digital downloads and groceries (depending on the country website). In addition to the basic buying and selling services, Amazon also offers social networking features to their users. These features allow them to interact with the website and with other users creating different types of social networks. In this section, we study the main social networking features of Amazon and the social networks that emerge from them.

Amazon users can carry out many activities on the website. Among them, Amazon allows its users:

- To join to user communities: Amazon allows its users to create and update a profile and share it with other users, joining to different communities. In addition to adding personal information and media content to their profile, users can create Listmania lists with the Amazon products they are interested in or Wish lists with the products they want from Amazon. Communities allow their members to talk between them via discussion forums. As in reviews, each post can be replied, rated and reported, but these rates are not considered to compute any user's rank. Users can also suggest products to 
their communities by tagging them with any topic that is considered interesting for the community. Besides, users can write So You'd Like to... guides to give recommendations to other users about products they may be interested in. Guides can be rated and reported.

- To write reviews about its products: any Amazon user can write a review about a product, no matter if he has purchased it or not. As part of their review, users can also rate products from 1 to 5 stars. Reviews for different media of the same product are grouped together (e.g. the review for a particular film, whether on VHS, Blu-Ray, or DVD, will include reviews from all three formats). Currently, there is no way to only look at reviews for one version of a product. Reviews can be annotated with the name of the reviewer or his popularity as reviewer (i.e. reviewer rank, starting from the best reviewer in rank 1). Reviewer ranks are not only computed by using the positive votes received, but also the number of negative votes and the time when the review was published. In addition, other users can write comments on reviews, rate them as useful/unuseful and report them to the company if they consider them offensive or inappropriate.

- To leave feedback about sales: after each purchase, buyers are offered the opportunity to leave feedback about the seller, rating the transaction from 1 to 5 stars (i.e. worst to best) and also writing a comment. Seller ratings (shown as percentages where $100 \%$ represents an excellent seller) are computed by using the votes received over the transactions performed in a specific period of time. Then, sellers have the opportunity of responding, but they cannot rate buyers (they can rate the transaction and this is displayed on the website, but only feedback submitted by buyers is considered to compute a seller rating).

These activities provide Amazon users with social networking features that allow them to interact with the website and between them. As a result of these interactions, social relations emerge and users form social networks, which will be analysed in the next section. Moreover, the Amazon website runs a powerful recommendation algorithm that matches each user's purchased and rated item to similar items, and outputs a personalised recommendation list [21]. This algorithm follows an item-to-item collaborative filtering approach that scales to massive data, produces high quality recommendations in real time and shows why items are recommended (usually because users bought a similar item).

Depending on the activities that users perform in Amazon, different user roles can be identified. Figure 1 shows a use case diagram with the roles that take part in the Amazon social networking features and the activities that users can carry out when they are playing each role. Note that users can play different roles when they are carrying out different activities on the website. In addition to them, Amazon users can play other roles (e.g. administrators, developers, associates, etc.) and perform other operations using other features (e.g. Amazon Web Services, Amazon Marketplace Payments, etc.), but they are out of the scope of this paper.

The most frequent type of user is a potential buyer that checks the website searching for a specific product. For clarity purposes, we consider this role as the default role that any user that registers on the website plays. Furthermore, specific users can also play the seller, author and publisher roles, depending on their business or their relation with the product. As explained above, after a purchase, the buyer has the opportunity of leaving feedback about his experience with the seller, reviewing the transaction. Apart from selling their products, sellers can also answer to this feedback if they want to add any comment or reply. In addition, Amazon allows authors and publishers to write comments on their works.

Other important roles from the social networking perspective is the reviewer role, which is played by any Amazon user as soon as he writes a review on any product or a comment on a review, sharing his knowledge and opinions about the product and making recommendations to other Amazon users. Note that we consider that users play the reviewer role both when they start a new review and also when they make comments on a review written by other users. Finally, any Amazon user is able to rate a review as useful/unuseful or report a review to the website if it is considered inappropriate.

\section{Social Network Model}

As pointed out before, Amazon allows its users to interact and form social networks. We have identified two types of social networks in the website: explicit, which are those formed by users that join to the same community or implicit, which emerge from writing reviews and from sales and their subsequent feedback. Although in communities users can engage in discussion forums and share content and preferences with other users, we consider that most of these activities are leisure oriented. Hence, the application of 


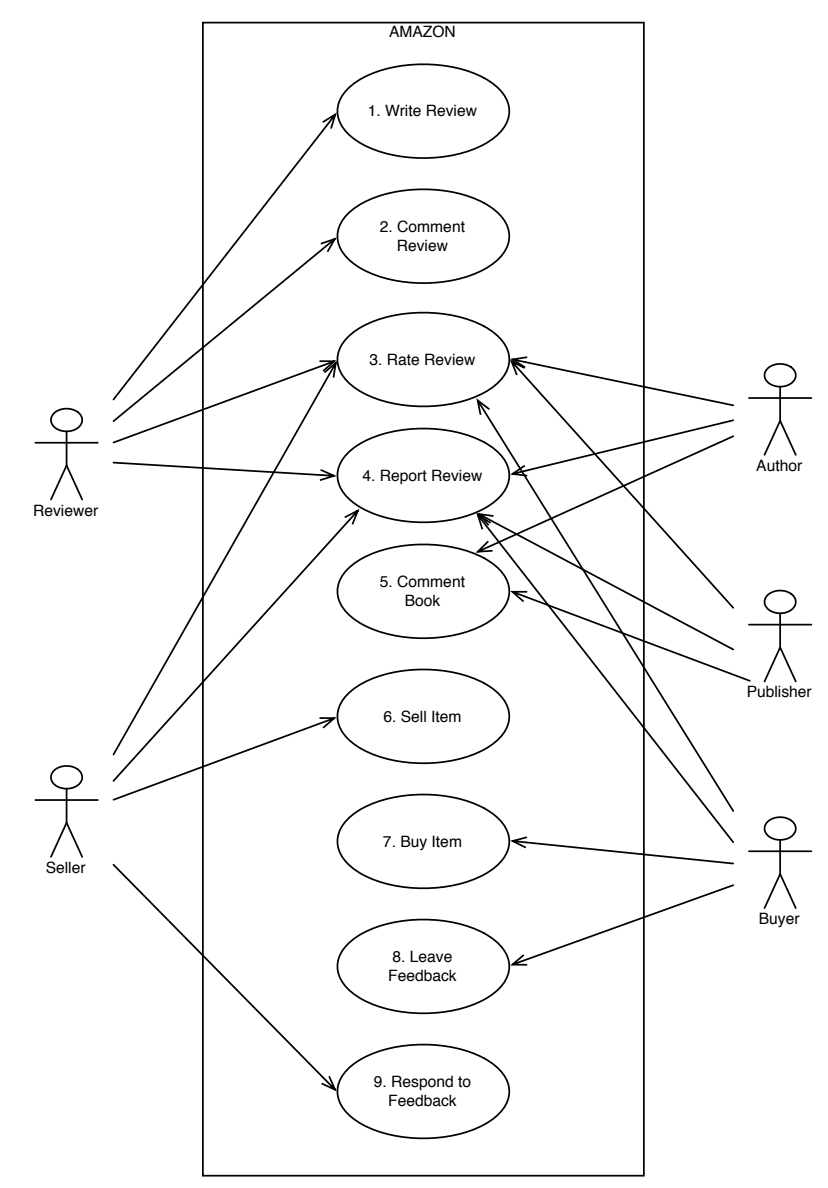

Fig. 1 Amazon Use Case Diagram

argumentation as a new technique to enhance the results obtained from the arguments that could appear in their underlying explicit social networks is less important and obvious than in the implicit social networks that reviews and sales entail. We focus then on analysing the social network model that emerges from reviews and sales following our social network abstraction.

\section{Social Network of Reviews}

The reviews on products that Amazon users write give rise to a social relation between them. From this relation emerges a social network that we have called Amazon Social Network of Reviews. The tasks permitted on the network are: writing reviews on Amazon products, writing comments on reviews, rating reviews or reporting reviews. Following Walton and Krabe's typology, the main type of dialogue enabled by this social networking activity is information seeking and sharing, since the overall purpose of the network is such of making reviews on Amazon products, recommending them or advising other users against. However, comments on reviews can add more information, amend the original text or even provide opposing opinions. In that case, persuasion dialogues can also be held.

Figure 2 left is an example of a network of reviews with six Amazon users playing each role that is involved in the activity of writing reviews. Arrows from User 1 to Users 3, 4, 5 and 6 stand for social ties meaning that User 1 has written a review about a product related with those other users (because they are sellers, authors, publishers or buyers of the product). Arrows from Users 3, 4, 5 and 6 to User 1 stand for a social tie that represents that those users rated or reported the review of User 1 . Finally, arrows between Users 1 and 2 represent social ties that can be created when reviewers comment, rate or report the review of other reviewers. Also, comments on reviews can also be commented, rated and reported.

In the figure, knowledge databases are attached to each role. With them, we represent the information that the website stores for each role. Thus, the reviews made by a reviewer and how they were rated 

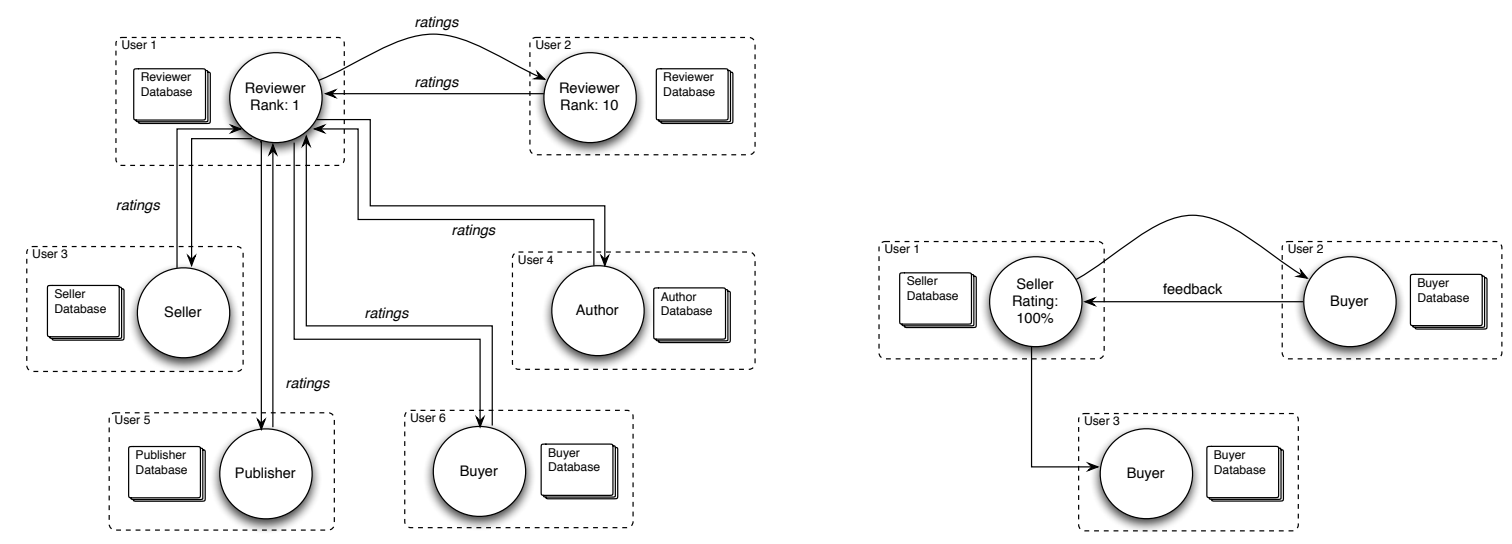

Fig. 2 Left: Amazon Social Network of Reviews; Right: Amazon Social Network of Sales

would be stored in the reviewer's database. However, it is not clear which information is really stored in Amazon databases, since not all is available to be checked on-line by its users. For instance, there is no way for a user to check the list of reviews that he has rated or reported. Nevertheless, these activities give rise to social relations between the users involved in them and hence, we take the information that they could produce into account to perform our analysis.

Regarding social network analysis measures, Amazon uses several measures to evaluate its users that can also be used as metrics to evaluate their performance in the social network. For instance, the reviewer's rank can be used as a reputation measure to evaluate the importance of a specific node (representing a reviewer) in the social network of reviews. In addition, other measures could be used to label ties. For instance, individual trust measures about specific reviewers could be computed for each user by using the percentage of useful ratings that the user has assigned to each reviewer over a certain period of time.

\section{Social Network of Sales}

The other central activity that enables a social interaction between Amazon users is the sales performed on the website. We capture this interaction by using the abstraction of an Amazon Social Network of Sales. In this case the overall purpose of the network is to run commercial transactions between its members and to inform about them. Therefore, the tasks permitted on the network are sell and buy products and leave feedback about these commercial transactions. The main type of dialogue that these tasks enable is that of information seeking and sharing, since by leaving feedback a buyer shares his sales experiences with the website administrators and other users. In addition, this feedback can be replied to by the seller. Thus, sellers have an opportunity to leave their arguments and persuade other users to mistrust buyers that have reported unfair feedback.

Figure 2 right shows an example of an Amazon social network of sales with one node representing a user playing the role of a seller (i.e. User 1) and two more nodes representing users that play the role of buyers of the products sold by this seller (i.e. Users 2 and 3). The arrow from User 1 to Users 2 and 3 means that this user has sold a product to Users 2 and 3 and thus, a social tie between them has been created. The arrow from User 2 to User 1 represents that User 2 has provided feedback about his sale with User 1 and hence, this has generated a new social tie. This arrow can be labelled with the numeric feedback (ranging from 1 to 5 stars) that the buyer has left about the transaction, which we consider as a social network analysis measure. Amazon aggregates these numbers to compute a seller rating, which can also be used as a reputation measure to label nodes that represent users playing the role of sellers.

In addition, as pointed out before, the seller can also leave comments about the transaction and the feedback received (although not about the buyer himself and hence, this assessment does not count towards any rating about him). This is represented in the figure by the arrow from User 1 to User 2 . Thus, this arrow cannot be labelled with a feedback score. Finally, we have represented in the figure the information that Amazon stores for sellers and buyers by their respective databases. 


\subsection{Missing Social Networking Features}

Previous sections have analysed the main social networking features of Amazon, showing the structure and operation of their underlying social networks of reviews and sales. From this analysis we can conclude that the main activities that users can perform on Amazon website (i.e. writing reviews, making sales and leaving feedback and joining communities) show most of the features that usual social networking activities have, but still some are missing.

\section{Community Services}

Amazon community services include almost all common social networking features, except for chat. In fact, these websites do not provide an on-line chat service, probably because this feature is more usual in leisure oriented social networks and its addition could hinder the business objective of Amazon.

Amazon users can create and update a user profile, where friends and other people can find a specific user and learn more about him. From the profile users can access and manage their community content, recent purchases, reminders, friends and other people on Amazon. The profile also shows how people are voting on the user's reviews, images, Listmania lists and guides. Furthermore, users can share messages with other users participating in forums, form groups, tag and suggest interesting products for the community and rate and report posts.

\section{Writing Reviews}

Regarding the reviewing activity, the information about Amazon reviewers can be browsed in their user profile. However, apart from the profile, Amazon only enables two additional social networking features for this activity: sharing messages with other users (i.e. the reviews themselves) and providing rates and reports about them.

Thus, Amazon does not allow users playing the reviewer role to keep a list of friends with, for instance, users with reviews on the same items, preferred reviewers, etc. Reviewers cannot join groups of specialised reviewers, who could make intensive reviews on a specific type of items and agree tags and recommendations for other users. In addition, another interesting feature that these websites lack could be to provide users with easy access from their profile to the information about reviewers and reviews that they could find interesting. A possible way to do so would be to provide a direct link to the profile of reviewers that have made a review on an item of interest to the user (e.g. he has made a review on the same item, he has participated in a sale about this item or he has shown interest in the item). The addition of these social networking features would entail to make explicit the social network of reviews and allow users to contact with their neighbours in the network. To date, the user can only reach part of this information by making searches for items in the website and looking for the attached reviews, which is quite time consuming and uncomfortable.

\section{Sales and Feedback}

In the case of sales, Amazon keeps a sellers' account associated to each user account. This account includes a seller's profile, which can be browsed by users to read the feedback and comments that other users have left, check the seller's rating and browse other items offered by the seller (i.e. shared content). Although sellers can comment upon the feedback that they have received, they cannot leave feedback from buyers. Thus, Amazon does not provide a specific buyers' profile. The company has taken this policy to encourage users to leave true feedback, not worrying about being punished by sellers if they do not leave good feedback about them. However, it could be very interesting for sellers to be able to check a buyers' rating before making business with them. This could be implemented by following a policy of allowing sellers to leave only positive ratings about buyers. In addition, sellers and buyers can share messages and report irregularities in sales. However, these messages are only accessible from the sellers' profile or the user profile of the buyers during certain period of time (depending on the country website).

By making explicit its network of sales, Amazon could add interesting social networking features for buyers and sellers. In this way, sellers could keep a list of friends with regular buyers or join groups with sellers of similar items. Similarly, buyers could easily contact other buyers of the same products to share opinions about items and sellers. These features would improve the current way for sellers and buyers to 
contact other users with similar interests by browsing discussion forums in Amazon Communities. However, the easy access to all information about sales would increase the competence. Differences among the quality of sellers, prices, etc. would be clearly available for every user, which could have an undesirable effect for Amazon business.

\section{Research Challenges for Argumentation in Social Networks}

The analysis performed in the previous sections shows how one of the main current business oriented websites, Amazon, provide its users with very similar social networking features. By means of the activities that these features entail, users form different types of social networks. Also, these networks enable dialogues among their users, which give rise to a great amount of important but often unstructured information. To this point in the paper, we have analysed the reviews and sales features of Amazon, characterising the underlying interactions among their users by means of social network abstractions. However, although space restrictions do not allow us to include further analysis on other business, decision support or leisure oriented websites, it is easy to check how most of them offer the same features to their users and an analysis can lead to the same conclusions. Thus, results shown here are applicable and transferable to other similar websites.

Based on the analysis performed so far, we have identified in this section interesting future research opportunities for argumentation in social networks. Also, we advance research on applying argumentation techniques to enhance the performance of the emergent activities carried out by the users of a social network.

\subsection{Open Research Challenges}

In addition to the contributions motivated by the related work cited in Section 2, we have identified the following open research challenges for argumentation in social networks:

- Suggesting acquaintances: A typical recommendation feature that almost every social networking website includes is a friends recommender. To date, websites suggest potential acquaintances to the users of their social networks by using similarity measures among their profiles (e.g. similar preferences, common friends). In a world where social capital and privacy are gaining increasing importance, to receive justifications and support to evaluate the usefulness and suitability of acquiring new acquaintances before just adding them to one's contact list would be very interesting.

- Argumentation for ontology disambiguation and alignment: The growing number of ontologies that represent the same concepts with different terms gives rise to the need of tools for determining correspondences between these concepts (e.g. ontology alignment). Also, the other way around, the same term can have different semantics in different ontologies and thus, mechanisms for performing the semantic disambiguation are necessary. However, up to this point in the paper, we have spoken about Web 2.0 related issues, which have been argued to be opposing to the Semantic Web approach. There has been considerable debate between those who favour the formality of controlled vocabularies and ontologies of Semantic Web and those who prefer the more informal nature of social tagging (i.e. collaboratively creating and managing tags), inherent to the Web 2.0 applications [2]. Nevertheless, we share the ideas shown by [23] that argues that despite the machine-orientation of the Semantic Web, the process of creating and maintaining it is a social one, particularly in the creation and managing of ontologies. Thus, the application of argumentation to ontology alignment and disambiguation provides important advances in this area [19][36].

- Argumentation for social decision making: Many social networks have tools that allow an on-line dialogue among users to search for information and support to make decisions on a wide range of topics. Argumentation has been successfully applied as a method to decision making with a powerful explanatory power [1]. In addition, it can be also used for explaining a choice once it has been made. Argumentation-based models for decision making would be adapted to social domains and used to enhance the decision of a social network user, for instance, by looking to the emergent behaviour of other users of his network. 


\begin{tabular}{|c|c|c|c|c|c|c|c|}
\hline Activity & Persuasion & Negotiation & Inquiry & Deliberation & $\begin{array}{c}\text { Information } \\
\text { Seeking and } \\
\text { Sharing }\end{array}$ & Eristics & Examples \\
\hline$\overline{B l o g s}$ & $\mathrm{x}$ & & & & $\mathrm{x}$ & & Pingback, Slashdot, LiveJournal, BlogSpot \\
\hline $\begin{array}{l}\text { Collabora- } \\
\text { tive RT } \\
\text { Editors }\end{array}$ & & & & & $\mathrm{X}$ & & $\begin{array}{l}\text { SubEthaEdit, SynchroEdit, ACE, Moonedit, Google } \\
\text { Docs \& Spreadsheets and Zoho }\end{array}$ \\
\hline $\begin{array}{l}\text { Commercial } \\
\text { Sites }\end{array}$ & $\mathrm{x}$ & & $\mathrm{x}$ & $\mathrm{x}$ & $\mathrm{x}$ & $\mathrm{x}$ & Amazon and eBay \\
\hline $\begin{array}{l}\text { Commercial } \\
\text { Social } \\
\text { Networks }\end{array}$ & & $\mathrm{X}$ & & & $\mathrm{X}$ & & Dell IdeaStorm \\
\hline $\begin{array}{l}\text { Consumer } \\
\text { Review } \\
\text { Sites }\end{array}$ & $\mathrm{X}$ & & $\mathrm{X}$ & $\mathrm{X}$ & $\mathrm{X}$ & $\mathrm{X}$ & TripAdvisor and ePinions \\
\hline $\begin{array}{l}\text { Deliberative } \\
\text { Social } \\
\text { Networks }\end{array}$ & $\mathrm{x}$ & & & $\mathrm{x}$ & & $\mathrm{x}$ & $\begin{array}{l}\text { Webs of discussion and debate for decision-making } \\
\text { purposes between individuals and government }\end{array}$ \\
\hline $\begin{array}{l}\text { Social help } \\
\text { Sites }\end{array}$ & $\mathrm{X}$ & & $\mathrm{X}$ & $\mathrm{x}$ & $\mathrm{X}$ & $\mathrm{X}$ & Kiva and Ethicaleconomy \\
\hline Forums & $\mathrm{x}$ & & & $\mathrm{x}$ & $\mathrm{x}$ & $\mathrm{x}$ & Yahoo! Groups and Google Groups \\
\hline $\begin{array}{l}\text { Instant } \\
\text { Messaging }\end{array}$ & & & & & $\mathrm{x}$ & & $\begin{array}{l}\text { Gtalk, Skype, ICQ, Yahoo! Messenger, MSN, Pidgin } \\
\text { AOL and Jabber }\end{array}$ \\
\hline $\begin{array}{l}\text { Prediction } \\
\text { Markets }\end{array}$ & & & $\mathrm{x}$ & & $\mathrm{x}$ & & \\
\hline $\begin{array}{l}\text { Social } \\
\text { Cataloguing }\end{array}$ & & & $\mathrm{x}$ & & $\mathrm{x}$ & & CiteULike, Connotea, BibSonomy and refbase \\
\hline $\begin{array}{l}\text { Social } \\
\text { Guides }\end{array}$ & & & & & $\mathrm{x}$ & & WikiTravel \\
\hline $\begin{array}{l}\text { Social } \\
\text { Libraries }\end{array}$ & & & $\mathrm{x}$ & & $\mathrm{x}$ & & discogs.com, imdb.com and LibraryThing \\
\hline $\begin{array}{l}\text { Social } \\
\text { Network } \\
\text { Search } \\
\text { Engines }\end{array}$ & & & & & $\mathrm{X}$ & & Newstrove \\
\hline $\begin{array}{l}\text { Social } \\
\text { Networking } \\
\text { Sites }\end{array}$ & $\mathrm{X}$ & & & $\mathrm{X}$ & $\mathrm{X}$ & $\mathrm{X}$ & Facebook, Flickr, MySpace, Orkut and Twitter \\
\hline $\begin{array}{l}\text { Social } \\
\text { On-line } \\
\text { Storage }\end{array}$ & & & & & $\mathrm{x}$ & & Using servers or P2P technology \\
\hline Text Chat & & & & & $\mathrm{X}$ & & IRC and other technologies \\
\hline $\begin{array}{l}\text { Virtual } \\
\text { Worlds }\end{array}$ & $\mathrm{X}$ & $\mathrm{X}$ & $\mathrm{X}$ & $\mathrm{X}$ & $\mathrm{X}$ & $\mathrm{x}$ & $\begin{array}{l}\text { Dotsoul, SecondLife, Active Worlds, the Sims on-line, } \\
\text { There, Planeshift, Croquet project, VOS, Solipsis, } \\
\text { Everquest and World of Warcraft }\end{array}$ \\
\hline Wikis & & & $\mathrm{X}$ & & $\mathrm{X}$ & & Wikipedia, Wikisource \\
\hline
\end{tabular}

Table 1 Argumentation dialogues enabled by social networking activities.

- Studying the properties and structure of on-line dialogues: We have performed a preliminary analysis over some of the activities that different types of argumentation dialogues enable (mainly extracted from the list of social software cited in Wikipedia ${ }^{9}$ ) that has resulted in the classification shown in Table 1. A potential application of argumentation in social networks is the study of the properties of such dialogues. This research can clarify the dynamics of the line of reasoning that follows each on-line debate and provide users with a formal way of evaluating and attacking the opinions of other users.

Advancing research on the last open issue, in the next section we propose the application of the argumentation schemes theory to formalise the underlying reasoning shown in the dialogues held among the users of the network (writing and commenting reviews and leaving and replying to sales feedback in our cases of study).

\subsection{Argumentation schemes in on-line dialogues in Social Networks}

As pointed out in section 1, argumentation schemes are stereotyped patterns of human reasoning that can improve the user's understanding about discussions and provide a means to evaluate what users have stated and why [40]. They are characterised by a set of premises and their underlying conclusion. Moreover, argumentation schemes have attached a set of critical questions (CQs) that stand for potential attacks that could refute the conclusion drawn from the scheme. This feature is very useful to guide argumentation dialogues. Thus, if a proponent of a position uses a pattern of reasoning that matches with an argumentation scheme, an opponent can try to pose one of its critical questions to attack the position of the proponent.

As a result of the analysis performed in the previous sections, we can identify the following advantages of applying argumentation schemes to formalise interaction dialogues on social networks:

9 Wikipedia: Social software. http ://en.wikipedia.org/wiki/Social_software 


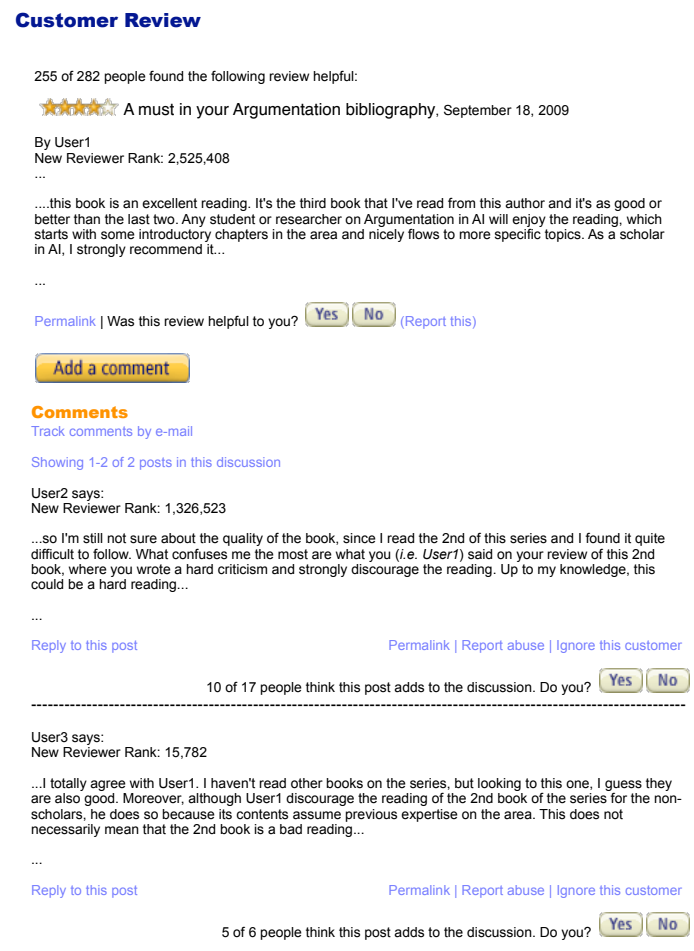

Fig. 3 An example on Amazon reviews

- To provide a formal structure to individual user opinions and recommendations, clarifying the reasoning patterns that have been followed to come up with them. This allows for explanations and justifications that clarify the position of the user that has posed them and prevent users from being misunderstood.

- To provide an objective way of evaluating user opinions and recommendations by looking at their associated reasoning patterns. This also clarifies the possible ways of attacking them by checking the critical questions attached to argumentation schemes.

- To provide a formal structure to the dialogue, improving the user's understanding about its underlying reasoning process. This clarifies the contributions and opinions of each individual user.

To demonstrate these advantages we have studied several user reviews and feedback comments on Amazon, providing here an example ${ }^{10}$ of how they could be formalised by using argumentation schemes. Therefore, let us present the conversation extract shown in figure 3 between three Amazon users that belong to the same social network of reviews. In this conversation, we can identify several arguments that encourage or discourage the reading of a book B. First, User1 provides an argument for encouraging the reading of the book that can be summarised as:

\section{A1:}

I am a scholar in the area of AI

I strongly recommend the reading of the book

So, this is a good reading

Then, the comment of User2 replies to this post by stating two arguments, one that gives his opinion about the topic and another that supports this view by attacking the argument of User1:

A2:

I have read the 2 nd book of the series of $B$

This wasn't a good reading

So, book $B$ couldn't be a good reading either

A3:

10 This example is an adaptation of real posts on Amazon. 
User1 says that book $B$ and its series are good

User 1 posted a hard criticism and discouraged the reading of the book $B$ in a previous review

So, the review of User1 is inconsistent with what he said previously

Finally, the comment of User3 replies to the comment of User2 by providing an argument that supports the argument of User1:

\section{A4:}

User1 is a scholar in the area of AI

User 1 discourages the reading for non-scholars of the 2 nd book of the series of $B$

So, the reading of the 2 nd book of the series of $B$ isn't a good reading for non-scholars

These arguments follow the pattern of reasoning stereotyped in some of the argumentation schemes listed in [40]. For instance, they could be translated into argumentation schemes as:

\section{A1: Argument From Expert Opinion}

Major Premise: Source User 1 is an expert in subject domain AI containing proposition book $B$ is a good reading Minor Premise: User 1 asserts that book $B$ is a good reading is true

Conclusion: book $B$ is a good reading is true

CQ1: How credible is User1 as an expert source?

CQ2: Is User1 an expert in the field $A I$ that the proposition book $B$ is a good reading is in?

CQ3: What did User1 assert that implies that book $B$ is a good reading?

CQ4: Is User1 personally reliable as a source?

CQ5: Is the proposition book $B$ is a good reading consistent with what other experts assert?

CQ6: Is User1's assertion based on evidence?

\section{A2: Argument From Position to Know}

Major Premise: Source User2 is in position to know about things in a certain subject domain books on $B$ series containing proposition book $B$ is a good reading

Minor Premise: User2 asserts that book $B$ is a good reading is false

Conclusion: book $B$ is a good reading is false

CQ1: Is User2 in position to know whether book $B$ is a good reading is true of false?

CQ2: Is User2 an honest source?

CQ3: Did User2 assert that the book B is a good reading is true or false?

\section{A3: Argument From Inconsistent Commitment}

Initial Commitment Premise: User1 has claimed that he is committed to proposition book $B$ and its series are a good reading

Opposed Commitment Premise: Other evidence in this particular case shows that User1 is not really committed to proposition book $B$ and its series are a good reading (since he posted a hard criticism and discouraged the reading of the book $B$ in a previous review)

Conclusion: User1's commitments are inconsistent

CQ1: What is the evidence supposedly showing that User1 is committed to proposition book $B$ and its series are a good reading?

CQ2: What further evidence in the case is alleged to show that User 1 is not committed to proposition book $B$ and its series are a good reading?

CQ3: How does the evidence from premise 1 and premise 2 prove that there is a conflict of commitments?

\section{A4: Argument From Expert Opinion}

Major Premise: Source User1 is an expert in subject domain AI containing proposition book $B$ is a good reading

Minor Premise: User1 asserts that the 2 nd book of the series of B isn't a good reading for non-scholars is true

Conclusion: the 2nd book of the series of B isn't a good reading for non-scholars is true

(Same CQs than A1)

Therefore, by making the identification of the schemes that each argument fits, we are able to provide a formal structure to user opinions. This structure clarifies the pattern of reasoning that the arguments follow. In this way, they can be evaluated objectively and users can be asked for justifications in a structured way. Thus, any critical question attached to these schemes could be used to ask the user for an explanation of his assumptions or either to attack the argument that the scheme represents, thus providing an exception that rebuts its conclusion. For instance, in this example argument A3 poses an attack on argument A1 by instantiating a critical question of the Argument From Expert Opinion (see [40] for the full set of critical questions) that also fits the scheme of the Argument From Inconsistent Commitment:

A3 attacks A1 instantiating its CQ4: Trustworthiness Question: Is User1 personally reliable as a source? assuming that a person with inconsistent commitments, as stated by A3, is not personally reliable as a source

Moreover, A4 attacks A3 by instantiating a critical question of the Argument From Inconsistent Commitment that invalidates its conclusion and thus, implicitly supports A1: 
A4 attacks A3 instantiating its CQ2: What further evidence in the case is alleged to show that User1 is not committed to book $B$ and its series being a good reading?

by distinguishing the fact of being committed to book $B$ and its series are a good reading from the fact of being committed to the 2nd book of the series of B isn't a good reading for non-scholars

As shown, the identification of the argumentation schemes of each argument also provides a formal structure to the dialogue, clarifying what has been the contribution of each user and what his objective is in posting such specific opinion. Note that we assume that this is an extract of a larger dialogue between several users. Thus, although the line of reasoning here could seem obvious, each individual opinion could be blurred as the number of posts grows. This example shows how attaching argumentation schemes to user opinions in the dialogues held in social networks a) clarifies the positions of each user and the line of reasoning that follows their arguments, avoiding misunderstandings; b) clarifies the possible ways of supporting/attacking these arguments and c) provides a clear structure for the full dialogue.

In addition, further features of the social network could be also used to produce arguments and attacks. For instance, in this example a low reviewer rank could be used to attack A1 by instantiating its CQ1 Expertise Question: How credible is User1 as an expert source, assuming that this rank stands for the reputation as reviewers of the users of the network. Note that although in the example attacks also match with other argumentation schemes, this does not mean that other kinds of information couldn't be used as arguments. Nevertheless, other argumentation schemes could also fit these opinions (note that the same argument could fit several argumentation schemes). For instance, A1 could also fit the Argument From Position to Know, since User1 states that he has also read the other books on $B$ series, so he could be thought of as being in a position to know about things in this subject domain. In this case, the author of each post should choose the argumentation scheme that best fits his line of reasoning.

However, an important challenge for the real application and success of this approach is to facilitate to expert and non-expert users the identification of the argumentation schemes that fit the structure of their opinions. Next section provides insights in this regard.

\subsection{Schemes identification}

Argumentative elements in generic social media tools are very basic and indeed, argumentation support has not yet moved firmly from the academic lab [33]. If websites facilitates their users the identification of argumentation schemes and furthermore, if users are rewarded for that (for instance, by increasing some scoring of confidence on their opinions), the probability that users actually use this resource highly increases. Otherwise, most users will find it tough and useless to attach any reasoning scheme to their posts instead of just writing them.

If business oriented websites like Amazon want to foster the adoption of argumentation techniques in the on-line debates among the users of their social networks, tools for facilitating the identification of the arguments structure and related argumentation schemes are necessary. For users that have some experience with argumentation concepts, to provide a plug-in to mark premises and conclusions and classify them as parts of argumentation schemes could be enough. After that, this information could be stored in the website and subsequently processed and used. Thus, other users could browse the reasons that a user has for arguing that an item is bad or good, for providing specific feedback, etc. Moreover, those users could look the critical questions associated to argumentation schemes to try to rebut the original argument. A possible way to implement this plug-in could be to follow the approach of the Argument Blogging project, which allows to select pieces of text and mark them as arguments, supports and rebuts for arguments, or adapt a tool like the ones proposed in OVA, which provide a drag-and-drop interface for analysing textual arguments (see Section 2.4 for references about these tools). However, although these tools were available, users without experience in argumentation could find it difficult to identify the structure of their arguments or to select the argumentation scheme that best fits their pattern of reasoning. Thus, the website should facilitate users to attach arguments to their positions on the on-line dialogue. A possible way to do so would be to present the user with a set of questions whose answers could label parts of his argument or classify the associated argumentation schemes from a decision tree. For instance, asking the user if he considers himself an expert in a topic could guide the website to attach the Argument from Expert Opinion scheme to his position. Also, asking users to provide pieces of evidence of a specific type to rebut the argument of another user could help to attach other argumentation schemes, such as the Argument from Inconsistent Commitment or the Argument from Exception (see [40]) to their 
underlying arguments. In addition, the models and argumentation schemes recently proposed in [42] can also help the clarification and development of user-relative arguments to justify evaluative statements. However, the main drawback for the approach is the creation of this decision tree, which would entail to provide a classification and hierarchy over argumentation schemes. This is a demanding challenge that still has to be solved by the argumentation research community and is out of the scope of this paper. A first step to cope with it was proposed in [40].

\section{Conclusions and Future Work}

In this work, we have analysed open research issues for argumentation in social networks. Also, we have shown how argumentation schemes theory can provide a valuable help to formalize and structure on-line discussions and user opinions in decision support and business oriented websites. Thus, we have analysed the social networks that originate from these discussions in a real case, Amazon website. Commercial sites like them have exploited some features of the social networking concept, but not all. The possible reasons why and why not were discussed in section 4.2. As pointed out before, argumentation theory could provide value for the functions of eliciting, organizing, analysing, summarizing, resolving, and presenting information collected through social networks. Thus, we suggest here some guidelines for decision support and business oriented websites to improve on-line dialogues in social networks using argumentation. The following list does not pretend to be comprehensive, but just summarise and highlight some ideas and thoughts that have been developed along this work:

- Provide an explicit and illustrative way of viewing and accessing the ties and contacts that posts and answers to posts (and correspondingly feedback ratings and responses to feedback) give rise. Thus, by making explicit their underlying social networks, websites would allow users to exploit all information resources available in the website. In addition, this could enhance the trust and reputation that the website has from the users point of view.

- Provide easy-to-use tools for the quick and costless identification of argumentation schemes in the line of reasoning that a user is following in a post. These tools could be inspired by the actual tools for argument authoring and diagramming, as presented in section 5.3. Alternatively, the website could provide users with a simple questionnaire to fill in when they post their opinions, following the approach of [6]. However, the automatic identification could imply to develop complex tools for text analysis or, in the case of the questionnaire, it would imply to partially solve the open problem of providing a classification of argumentation schemes. Therefore, it could be easier to provide users with tools for making manually the identification and maybe, to grant some reward (e.g. positive feedback) to the users that user them.

- Provide tools for the easy identification of potential attacks to posts, by using the critical questions associated to their underlying schemes. Also, these tools could be similar to the above mentioned.

- Provide tools for summarising and analysing the information gathered from the schemes and attacks identification. This could help users to have a clear idea about what is being discussed, what are the positions of each user and how these positions are backed and rebutted. Also, users that support their positions and succeed in their attacks could be trusted as most experienced than others.

Acknowledgements This work summarises results of the authors joint research, funded by an STMS of the Agreement Technologies COST Action 0801, by the Spanish government grants [CONSOLIDER-INGENIO 2010 CSD2007-00022, and TIN2012-36586-C03-01] and by the GVA project [PROMETEO 2008/051]

\section{References}

1. L. Amgoud: Argumentation for Decision Making. Argumentation in Artificial Intelligence, pp. 301-320, Springer, 2009.

2. P. Anderson: What is Web 2.0? Ideas, technologies and implications for education. JISC Technology and Standards Watch report, 2007.

3. J. Bentahar, J-J. Ch. Meyer and B. Moulin: Securing Agent-Oriented Systems: An Argumentation and Reputationbased Approach. Proceedings of the 4th International Conference on Information Technology: New Generations (ITNG 2007), IEEE Computer Society, pp. 507-515, 2007.

4. S. Buckingham Shum: Cohere: Towards Web 2.0 Argumentation. Proceedings of the 2nd International Conference on Computational Models of Argument, COMMA, pp. 28-30, 2008. 
5. R. Burke: Hybrid Recommender Systems: Survey and Experiments. User Modeling and User-Adapted Interaction, Vol. 12, pp. 331-370, 2002.

6. D. Cartwright and K. Atkinson. Political engagement through tools for argumentation. In Proceedings of the Second International Conference on Computational Models of Argument (COMMA 2008), pp. 116-127, 2008.

7. C. Chesñevar, J. McGinnis, S. Modgil, I. Rahwan, C. Reed, G. Simari, M. South, G. Vreeswijk and S. Willmott: Towards an Argument Interchange Format. The Knowledge Engineering Review, Vol. 21, No. 4, pp. 293-316, 2006.

8. C.I. Chesñevar, A.G. Maguitman and M.P. González: Empowering Recommendation Technologies Through Argumentation. Argumentation in Artificial Intelligence, pp. 403-422, Springer, 2009.

9. A.J. García, J. Dix and G.R. Simari: Argument-based Logic Programming. Argumentation in Artificial Intelligence, pp. 153-171, Springer, 2009.

10. J. Golbeck: Generating Predictive Movie Recommendations from Trust in Social Networks. Proceedings of the Fourth International Conference on Trust Management, LNCS, Vol. 3986, pp. 93-104, 2006.

11. T. Gordon, H. Prakken, and D. Walton. The Carneades model of argument and burden of proof. Artificial Intelligence, Vol. 171, No. 10-15, pp. 875-896, 2007.

12. R. Guha, R. Kumar, P. Raghavan and A. Tomkins: Propagating Trust and Distrust. Proceedings of the 13th international conference on World Wide Web, pp. 403-412, 2004.

13. S. Heras, M. Navarro, Vicente Botti and V. Julián: Applying Dialogue Games to Manage Recommendation in Social Networks. Proceedings of the 6th International Workshop on Argumentation in Multi-Agent Systems, ArgMAS, 2009.

14. S. Heras, K. Atkinson, V. Botti, F. Grasso, V. Julián and P. McBurney: How Argumentation can Enhance Dialogues in Social Networks. 3rd International Conference on Computational Models of Argument, COMMA, Vol. 216, pp. 267-274, 2010

15. S. Heras, K. Atkinson, V. Botti, F. Grasso, V. Julián and P. McBurney: Applying Argumentation to Enhance Dialogues in Social Networks. ECAI 2010 workshop on Computational Models of Natural Argument, CMNA, pp. 10-17, 2010.

16. N. Karacapilidis and M. Tzagarakis: Web-based collaboration and decision making support: A multi-disciplinary approach. Web-Based Learning and Teaching Technologies, Vol. 2, No. 4, pp. 12-23, 2007.

17. D. Kim and I. Benbasat: Trust-related arguments in internet stores: A framework for evaluation. Journal of Electronic Commerce Research, Vol. 4, No. 2, 2003.

18. D. Kim and I. Benbasat: The Effects of Trust-Assuring Arguments on Consumer Trust in Internet Stores: Application of Toulmin's Model of Argumentation. Information Systems Research, Vol. 17, No. 3, pp. 286-300, 2006.

19. L. Laera, V. Tamma, J. Euzenat, T. Bench-Capon, and T. Payne: Reaching agreement over ontology alignments. In Proceedings of the 5th International Semantic Web Conference (ISWC 2006), 2006.

20. C. Lange, U. Bojãrs, T. Groza, J. Breslin and S. Handschuh: Expressing argumentative discussions in social media sites. Social Data on the Web (SDoW2008) Workshop at the 7th International Semantic Web Conference, 2008.

21. G. Linden, B. Smith and J. York: Amazon.com Recommendations: Item-to-Item Collaborative Filtering. IEEE Internet Computing, Vol. 7, No, 1, pp. 76-80, 2003.

22. G. Linden, J. Hong, M. Stonebraker and M. Guzdial: Recommendation Algorithms, Online Privacy and More. Communications of the ACM, Vol. 52, No. 5, 2009.

23. P. Mika: Ontologies are us: A unified model of social networks and semantics. Journal of Web Semantics, Vol. 5, No. 1, pp. $5-15,2007$.

24. M. Montaner, B. López and J.L. de la Rosa: Opinion-Based Filtering Through Trust. Cooperative Information Agents VI, LNCS, Vol. 2446, pp. 127-144, 2002.

25. S. Ontañón and E. Plaza: Argumentation-based Information Exchange in Prediction Markets. Proceedings of the 5th International Workshop on Argumentation in Multi-Agent Systems, ArgMAS, 2008.

26. M.J. Pazzani and D. Billsus: Content-Based Recommendation Systems. The Adaptive Web, LNCS, Vol. 4321, pp. 325-341, 2007

27. I. Rahwan, F. Zablith and C. Reed: Laying the Foundations for a World Wide Argument Web. Artificial Intelligence, Vol. 171, No. 10-15, pp. 897-921, 2007.

28. I. Rahwan and B. Banihashemi: Arguments in OWL: A Progress Report. Proceedings of the 2nd International Conference on Computational Models of Argument (COMMA), pp. 297-310, 2008.

29. C. Reed and D. Walton: Argumentation Schemes in Dialogue. In: Dissensus and the Search for Common Ground, OSSA-07, Volume CD-ROM, pp. 1-11, 2007.

30. J. Sabater and C. Sierra: Reputation and Social Network Analysis in Multi-Agent Systems. Proceedings of the 1st international joint conference on Autonomous agents and multiagent systems, No. 1, pp. 475-482, 2002.

31. J.B. Schafer, J.A. Konstan and J. Riedl: E-Commerce Recommendation Applications. Data Mining and Knowledge Discovery, Vol. 5, pp. 115-153, 2001.

32. J.B. Schafer, D. Frankowski, J. Herlocker and S. Sen: Collaborative Filtering Recommender Systems. The Adaptive Web, LNCS, Vol. 4321, pp. 291-324, 2007.

33. J. Schneider, T. Groza and A. Passant: A Review of Argumentation for the Social Semantic Web. Semantic Web Interoperability, Usability, Applicability, IOS Press, 2012.

34. C. Tempich, H. S. Pinto, Y. Sure and S. Staab: An Argumentation Ontology for Distributed, Loosely-controlled and evolvInG Engineering processes of oNTologies (DILIGENT). Proceedings of the 2nd European Semantic Web Conference, ESWC, pp. 241-256, 2005.

35. S.E. Toulmin: The Uses of Argument. Cambridge University Press, Cambridge, UK, 1958.

36. C. Trojahn, P. Quaresma, R. Vieira and A. Isaac: Comparing Argumentation Frameworks for Composite Ontology Matching. Proceedings of the 6th International Workshop on Argumentation in Multi-Agent Systems, ArgMAS, 2009.

37. TruthMapping: http://truthmapping.com/.

38. F.E. Walter, S. Battiston and F. Schweitzer: A model of a trust-based recommendation system on a social network. Journal of Autonomous Agents and Multi-Agent Systems, Vol. 16, No. 1, pp. 57-74, 2007.

39. D. Walton and E. Krabbe: Commitment in Dialogue: Basic Concepts of Interpersonal Reasoning. State University of New York Press, 1995.

40. D. Walton, C. Reed and F. Macagno: Argumentation Schemes. Cambridge University Press, 2008.

41. S. Wells, C. Gourlay and C. Reed: Argument Blogging. Computational Models of Natural Argument, CMNA, 2009.

42. A. Wyner and J. Schneider: Arguing from a Point of View. Proceedings of the First International Conference on Agreement Technologies, 2012. 